Article

\title{
Design and Analysis of Novel Actuation Mechanism with Controllable Stiffness
}

\author{
Erivelton Gualter dos Santos (D) and Hanz Richter*(D)
}

Mechanical Engineering Department, Cleveland State University, 2121 Euclid Ave, Cleveland, OH 44115, USA; e.gualterdossantos@vikes.csuohio.edu

* Correspondence: h.richter@csuohio.edu

Received: 3 December 2018; Accepted: 6 February 2019; Published: 9 February 2019

\begin{abstract}
Actuators intended for human-machine interaction systems are usually designed to be mechanically compliant. Conventional actuators are not suitable for this purpose due to typically high stiffness. Advanced powered prosthetic and orthotic devices can vary their stiffness during a motion cycle and are power-efficient. This paper proposes a novel actuator design that modulates stiffness by means of a flexible beam. A motorized drive system varies the active length of the cantilever beam, thus achieving stiffness modulation. New large deflection formulation for cantilever beams with rolling contact constraints is used to determine the moment produced by the actuator. To validate the proposed solution method, an experiment was performed to measure large static deformations of a cantilever beam with the same boundary conditions as in the actuator design. The experiments indicate excellent agreement between measured and calculated contact forces between beam and roller, from which the actuator moment is determined.
\end{abstract}

Keywords: variable-stiffness actuators; human-assist devices; flexible beams; energy-efficient actuators

\section{Introduction}

The overall performance of motion devices found in industry, entertainment, rehabilitation, prosthetics, and general robotics is dictated by the capabilities and efficiency of the driving actuator. Due to the increasing number of ways in which actuators can work alongside humans, safety and energy considerations are pivotal to their development [1]. Variable-stiffness actuators (VSA) were introduced to offer a compliant mechanical interface and opportunities for energy optimization through control. Many promising VSA designs have been proposed, with low stiffness and small energy losses due to friction. VSA concepts have also enabled the development of lightweight actuator designs that present advantages for powered human-assist devices, such as orthoses, prostheses, and exoskeletons. Comprehensive reviews of variable-stiffness actuation through the use of compliant elements may be found, for instance, in References [2,3].

VSA designs, and variable impedance actuators more generally, may be classified in a number of ways. In terms of the means to achieve impedance variations, a classification was made in Reference [4] according to the presence of physical springs, dampers, or inertial elements. Active impedance systems do not rely on such physical elements, obtaining variable impedance effects solely by means of motor feedback control. On the other hand, inherent compliance, damping, or inertial designs include the corresponding physical elements fitted with a mechanism to modulate their respective parameters. As in this paper, designs may exist that cut across categories.

In terms of actuator power requirements, those reported in the literature are often classified as active or passive actuators. An active device relies on an external source of power (such as a battery) to operate a motor providing force or torque. On the other hand, a passive device produces forces from 
the action of spring or damping elements. When a motor or other powered device is used to adjust the damping or stiffness of a physical element, the design is referred to as semiactive or quasi-active. In this case, the required power by the adjustment motor is negligible in comparison with that corresponding to the actuator output.

Any given variable impedance actuator draws characteristics from the above classes. The Mechanical Impedance Adjuster (MIA) of Reference [5] is of the inherent compliance and damping type. The prototype consists of three separate actuators to adjust compliance, position, and damping, and it requires four sensors. In terms of power classification, the MIA is active since an externally powered motor delivers the main actuation forces. Successful applications of the MIA design in single and multiple degrees of freedom (DOF) have been reported [6,7].

An important subclass of inherent compliance designs is given by Series Elastic Actuators (SEA), designed to include an elastic element between the joint and an externally powered actuator. These designs offer fast impact-absorption response and are therefore safe for operation with humans. The spring operates as a mechanical low-pass filter, whereby elevated force peaks are rapidly absorbed by the actuator and not transmitted to the support. Physical damping elements can also be added [4], or active control techniques may be implemented to achieve damping-like behavior [8]. SEA design may be optimized with the goal of decreasing energy consumption by proper selection of the compliant element's stiffness [9]. Furthermore, optimization techniques may be used to select the motor and the mechanism parameters [10-12].

Active impedance control greatly reduces reliance on physical elements and the corresponding complexity [3]. This approach has been used and accepted for several decades in industrial robotics [13,14]. For instance, in Reference [15], the compliant motion of an industrial robot manipulator is mimicked measuring joint torques and employing them as feedback to manipulate control gains. Recent studies by groups such as the German Aerospace Center (DLR) and KUKA Roboter also feature active compliance designs. The Lightweight Robot (LWR3) [16,17] and other achievements in physical human-robot interaction [18,19] are further examples.

Active compliance, however, presents disadvantages when compared to physical compliance mechanisms. First, the creation of a compliance effect (virtual spring) from an intrinsically noncompliant actuator, such as an electric motor, involves power losses that are not present when energy is stored and released from a physical spring. This is because a positive Joule dissipation power is associated with any current, regardless of its direction. System operation also relies on sensors, which are susceptible to failure, and there is additional complexity associated with the implementation of a feedback control system. Active compliance actuators have been primarily aimed at ensuring safety for humans [20] rather than improving energy efficiency.

Variable impedance designs have entered the field of powered prostheses and human-assist devices. They have presented a variety of advantages for users, such as comfort and energy-consumption reduction. Historically, the majority of prostheses (transtibial and transfemoral) presented fixed or limited stiffness variations during gait motion. Recently, researchers [21] introduced a nonbiologically inspired compliant prosthetic limb to modulate stiffness. A stack of leaf springs was introduced to provide the required compliance. Actuator stiffness is modulated with feedback control, allowing high-speed motion with small energy costs. A similar approach was taken in a human-robot collaboration to perform an isometric bicep curl with load [22]. In Reference [23], a novel variable-impedance controller modulated by force sensing was introduced to operate a powered leg prosthesis with energy regeneration. Surplus power associated with portions of the gait cycle is regenerated and stored in supercapacitors. Near-normal gait at net-zero power consumption was demonstrated in level walking by an amputee. The above designs clearly cut across the categories proposed in Reference [4].

Other mechanisms were found in the literature that are capable of stiffness modulation. In References [24,25], an ankle-foot prosthesis with stiffness modulation was proposed. To achieve different ankle stiffnesses during ambulation gait, the prosthetic foot uses cam-based transmission and a variable leaf spring to regulate the torque curve. From the rotation of the ankle, a deflection of 
the leaf spring is created. The active length of the leaf spring is controlled by a motorized lead screw, which is also responsible for adjusting the stiffness of the spring. This design is semiactive, since a small amount of power is used to adjust stiffness, and the required resisting torque at the ankle is generated without an actuating motor. Simultaneously, the design combines the features of active impedance control and inherent compliance designs.

The VSA concept of this paper can be regarded as a combination of active-impedance and inherent-compliance design principles. Specifically, a physical compliant element is used to ensure the primary requirements of the actuator, such as intrinsic compliance, load bearing and efficient energy storage, and release its capabilities. At the same time, the actuator has controllable features to modulate stiffness and damping. As in the MIA, the new device modulates stiffness by controlling the active length of a leaf spring. However, unlike the MIA, the mechanism is far simpler, backdrivable, and does not rely on friction to regulate damping. The proposed variable-stiffness mechanism is intended for use with a regenerative electromechanical drive system containing an electric storage element such as supercapacitor. Its design allows energy to be stored in the beam and in the supercapacitor and it is based on a flexible beam capable of large deflections, where stiffness is modulated by varying the position of a contact roller that determines the active length of the beam. When integrated with the electromechanical drive, the proposed VSA corresponds to the semiactive category.

Large beam deflections have been studied for at least 70 years [26]. Since then, a variety of new of methods have become available to solve this problem with different degrees of complexity and accuracy. Finite-element analysis (FEA) is extremely accurate and applicable to complex loading conditions. However, its computational complexity is the highest [27] compared with other methods, such as the circle-arc method [28] and the elliptic integral method [29]. Another approach to solving this problem is to use a Pseudorigid-Body Model (PRBM) [30]. The approach of Reference [31] involves the numerical solution of elliptic integrals. The same initial problem formulation is used in this paper.

The remainder of the paper is organized as follows: Section 2 describes the design concept for the proposed actuator; Section 3 presents the static solution for the geometry and large beam deflections; Section 4 presents an experimental validation of the solution method; and Section 5 concludes and presents future work discussion.

\section{Design Concept}

The proposed VSA in this paper was designed for use between any two rigid links connected by a rotational DOF. The design is aimed at storing and releasing energy with high efficiency while providing controllability and keeping the weight and complexity small. The actuator provides a controllable joint torque by means of the variable stiffness accomplished with a flexible cantilever beam. A roller is mounted on a pulley-driven shuttle used to vary the point of application of the force on the beam. This changes beam stiffness and consequently controls the joint moment. Due to beam elasticity, the mechanism can store and return potential energy.

A small electric motor and a servo drive with regenerative electronics are used to shift the contact roller. A supercapacitor is used to deliver or recover the electric energy that is converted by the DC motor/generator from the elastic potential energy of the beam. Supercapacitors are better suited as power-storage elements than batteries due to their high power density and ability to accept large charging currents [23,32]. The control system for this actuator, and its energy storage and release capabilities, are described in Reference [32].

Figure 1 shows how the proposed VSA could be used in transfemoral prosthesis. A pair of twin swivelling rollers are used to deflect the cantilever beam, and roller position can be shifted by the motor-driven pulley system. As detailed below, a controllable knee moment is obtained by modulating roller position. With proper control, the design offers the opportunity to minimize energy consumption while maintaining normal gait patterns. 


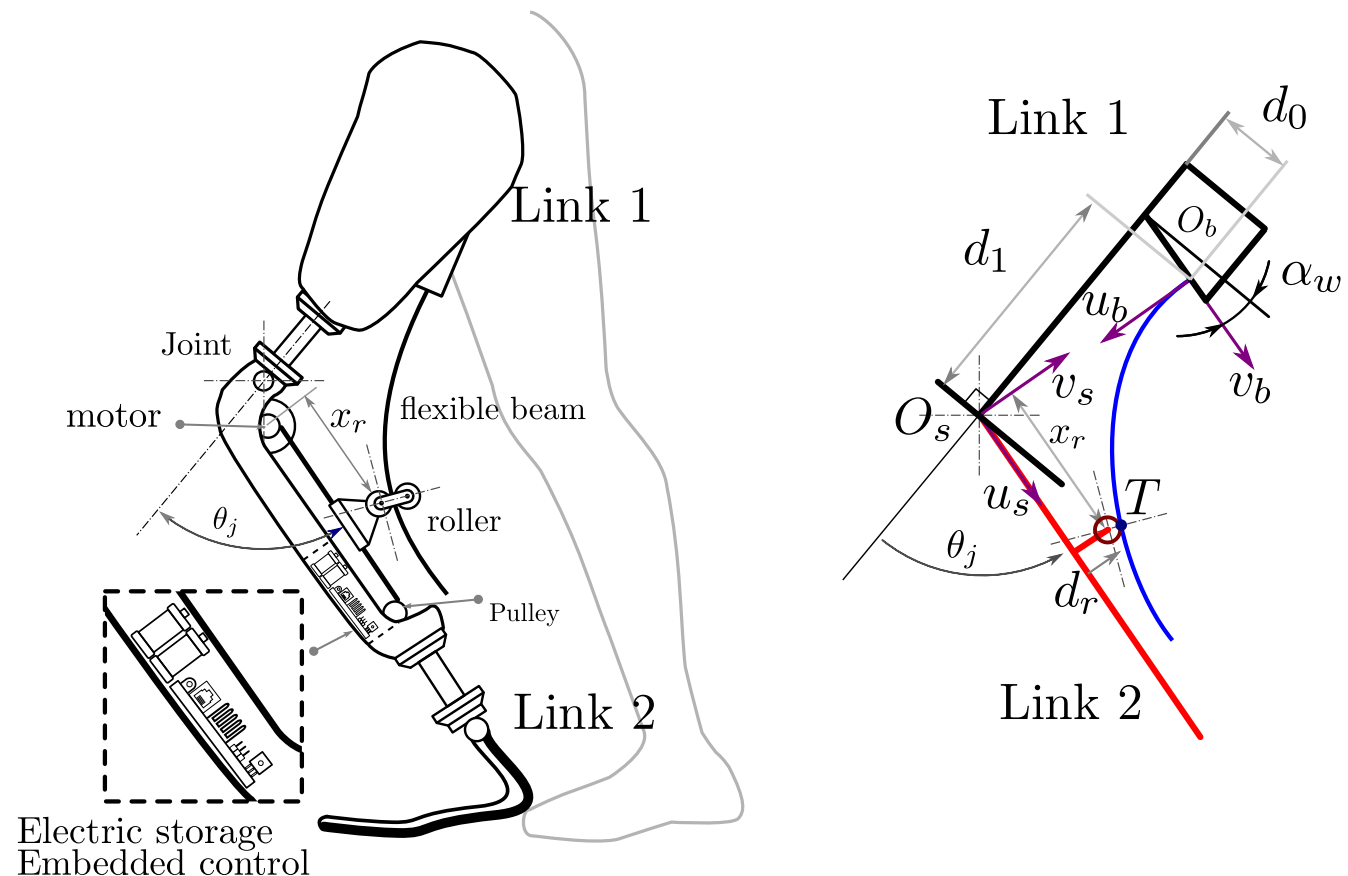

Figure 1. (a) General schematic of the proposed variable-stiffness actuator (VSA) design in a prosthetic leg application. (b) Geometry used for mechanism analysis.

Since rollers are not preloaded against the beam, we assume that contact occurs between the beam and only one of the rollers at a time. The corresponding reaction force can be decomposed into components perpendicular and parallel to the length of Link 2 (represented as the leg shank in Figure 1). The normal component creates a moment in the joint. The parallel component is a thrust force controlled with the motor-driven belt. When the beam releases energy, negative work associated with the thrust force is regenerated by the servo amplifiers and stored in batteries or supercapacitors. Conversely, power may be drawn from the electric storage to drive the belt, deflect the beam, and store elastic energy. Such bidirectional exchange of electric and elastic energy is expected to significantly contribute to overall system efficiency.

\section{Geometry and Beam Deflection Statics}

A desired knee-moment profile is obtained by suitably varying the point of application of force on the beam, which is, in turn, determined by knee angle and roller position. In order to study the use of the beam as a compliant mechanism, a cantilever beam, subject to large deflections, is examined. As shown in Figure 2, a co-ordinate frame $\left(u_{b}, v_{b}\right)$ was established to analyze deflections. Contact between beam and rollers occurs at single point $T$. Therefore, normal force $F$ must be perpendicular to both the beam and the roller in contact. Two component forces, $n P$ and $P$, are defined along the $u_{b}$ and $v_{b}$ axes, respectively. The beam is expected to undergo deflections exceeding the assumptions of elementary bending theory. That is, a given point on the beam is displaced in a plane, not merely in the $v_{b}$ direction. Euler-Bernoulli beam theory with geometric nonlinearity is suitable to analyze such cases whenever beam thickness is small relative to its width and length, so that shear deformations may be neglected.

Modeling large deflections in beams was extensively studied with different levels of complexity and accuracy [31]. However, the case under study introduces difficulty in that neither the active length of the beam nor the magnitude and orientation of the applied force are known a priori. Rather, these quantities become unknowns that appear in constraining equations associated with tangency with the roller. Therefore, only roller position $x_{r}$ and the angle between the links $\theta_{j}$ are known. Note that the cantilever beam is subjected to single-point force. For this study, this point is considered the end forces 
used in Reference [31], and it defines the active length of the beam. The following sections describe the proposed solution method that returns the contact-force components, the active beam length, and the end deflections and other variables of interest, such as the joint moment and elastic energy stored in the beam.

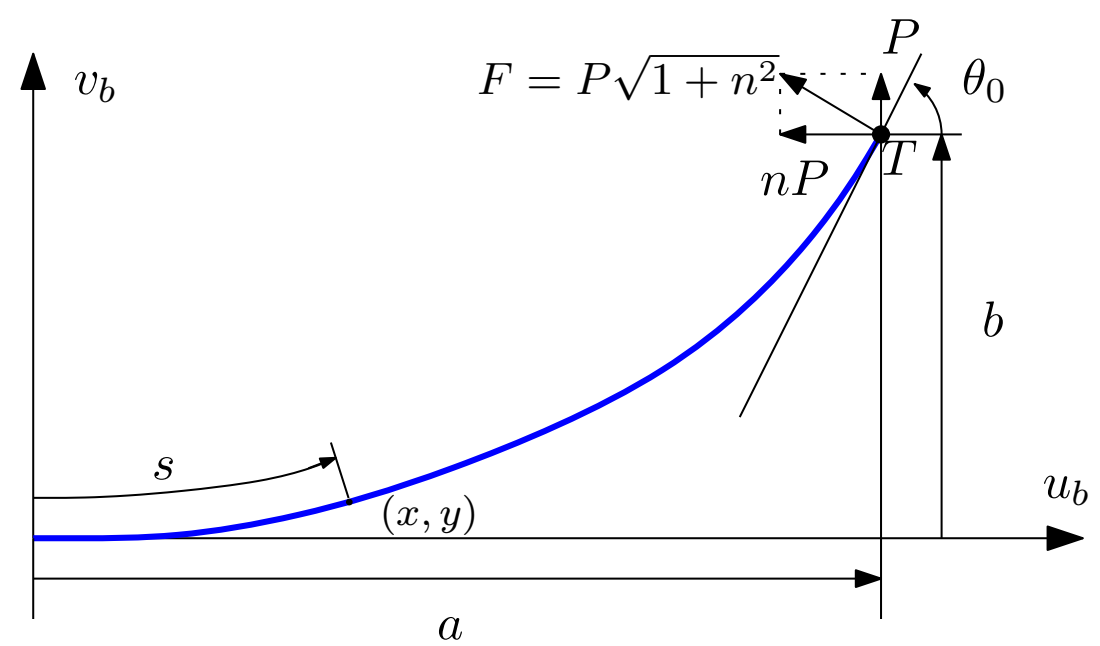

Figure 2. Geometry of beam deflection. Active length $L$ is the arc length between fixed end and point $T$.

\subsection{Large Beam Deflection Formulation}

The initial formulation of Reference [31] is used here, followed by a new approach to handle the unknown active length and applied force and orientation. The force applied at contact point $T$, as shown in Figure 2, is decomposed along the $\left(u_{b}, u_{v}\right)$ frame. These forces produce a moment at the origin of the frame that is related to beam geometry by the Euler-Bernoulli moment-curvature relationship:

$$
M\left(x_{b}, y_{b}\right)=E I \frac{d \theta}{d s}=P(a-x)+n P(b-y)
$$

where $\theta$ and $s$ are the beam slope and arc length, $E$ is the elastic module, and $I$ is the moment of inertia of the cross section. Additional geometric constraints due to the cantilever configuration determine a boundary-value problem:

$$
\begin{cases}\left.\theta\right|_{s=L} & =0 \\ \left.\frac{d \theta}{d s}\right|_{s=L} & =0\end{cases}
$$

where $L$ corresponds to the unknown active length of the beam. The solution of the nonlinear Equation (1) under boundary condition Equation (2), can be solved in terms of elliptic integrals:

$$
\begin{aligned}
& \frac{1}{\sqrt{2} \int_{0}^{\theta_{0}} \sqrt{\lambda-\sin (\theta)+n \cos (\theta) d \theta}}=\sqrt{\frac{2 P L^{2}}{E I}} \triangleq \alpha_{0} \\
& \frac{1}{\sqrt{2} \alpha_{0}} \int_{0}^{\theta_{0}} \frac{\cos (\theta) d \theta}{\sqrt{\lambda-\sin (\theta)+n \cos (\theta)}}=\frac{a}{L} \\
& \frac{1}{\sqrt{2} \alpha_{0}} \int_{0}^{\theta_{0}} \frac{\sin (\theta) d \theta}{\sqrt{\lambda-\sin (\theta)+n \cos (\theta)}}=\frac{b}{L}
\end{aligned}
$$

where $\lambda=\sin \left(\theta_{0}\right)-n \cos \left(\theta_{0}\right)$. Since $P, n$ and $L$ are unknown, additional constraints linking these quantities to $x_{r}$ and $\theta_{j}$ must be introduced.

\subsection{Rolling Contact Constraints}

A second coordinate frame $\left(u_{s}, v_{s}\right)$ is fixed at the joint with $u_{s}$ following the direction of the shank. A third frame $\left(u_{r}, v_{r}\right)$, parallel to the shank frame, is fixed at roller center $o_{r}$. In this frame, point $T$ is 
located at angle $\zeta$ measured from $v_{r}$, as shown in Figure 3. The roller is located at constant offset $d_{r}$ from the $u_{s}$ axis and at a variable distance $x_{r}$ from the origin along the $u_{s}$ axis.

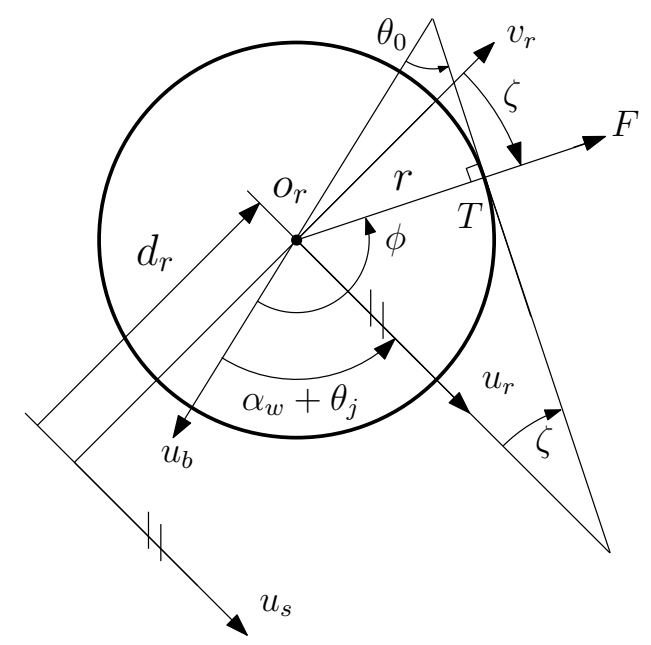

Figure 3. Rolling contact geometry constraints.

The joint angle is denoted as $\theta_{j}$ (angle between Links 1 and 2, assumed positive in flexion). The fixed end of the beam is at distances $d_{0}$ and $d_{1}$ from the joint, at a slant mounting angle $\alpha_{w}$, as shown in Figure 1.

Note that the slope of the beam at $T$ relative to $u_{b}$ is given by $\tan \left(\theta_{0}\right)$. Letting $\phi$ be the angle between the $u_{b}$ axis and the resultant force $F$, a straightforward derivation shows that the rolling contact constraint can be written as:

$$
\begin{aligned}
\phi-\theta_{0} & =\pi / 2 \\
\tan (\phi) & =-1 / n
\end{aligned}
$$

Moreover, it is straightforward to show that $\lambda=0$, and the following equation applies:

$$
\theta_{0}+\zeta=\alpha_{w}+\theta_{j}
$$

To relate end deflections $a$ and $b$ appearing in Equations (4) and (5) to the above quantities, a set of homogeneous coordinate transformations are defined following the notational conventions of robotics [33]. The transformation between frames $b$ and $s$ can be written as:

$$
H_{S}^{b}\left(\theta_{j}\right)=\operatorname{Rot}_{z, \alpha_{w}} \operatorname{Trans}_{x,-d_{1}} \operatorname{Trans}_{y,-d_{0}} \operatorname{Rot}_{z, \theta_{j}}
$$

and between frames $b$ and frames $r$ :

$$
H_{r}^{s}(u)=\operatorname{Trans}_{x, x_{r}} \operatorname{Trans}_{y_{,} d_{r}}
$$

The explicit form of the above transformation matrices is found in Appendix A.1. The coordinates of $T$ expressed in the $b$-frame can now be found as

$$
T^{b}=H_{s}^{b} T^{s}=H_{s}^{b} H_{r}^{s} T^{r}
$$

where $T^{r}$ contains the coordinates of $T$ in the roller frame, namely, $(r \sin (z), r \cos (z))$. A symbolic computation of the above transformations yields the pertinent expressions for $T^{b}=(a, b)$ :

$$
\begin{aligned}
& a=x_{r} \cos \left(\alpha_{w}+\theta_{j}\right)-d_{r} \sin \left(\alpha_{w}+\theta_{j}\right)-r \sin \left(\theta_{0}\right)-d_{1} \cos \left(\alpha_{w}\right)+d_{0} \sin \left(\alpha_{w}\right) \\
& b=x_{r} \sin \left(\alpha_{w}+\theta_{j}\right)+d_{r} \cos \left(\alpha_{w}+\theta_{j}\right)+r \cos \left(\theta_{0}\right)-d_{0} \cos \left(\alpha_{w}\right)-d_{1} \sin \left(\alpha_{w}\right)
\end{aligned}
$$


where Equation (8) has been used to eliminate $\zeta$. The above equations contain the unknown $\theta_{0}$, which must be determined from the beam-deflection relationships presented earlier.

\subsection{Numerical Solution Method}

Given $x_{r}$ and $\theta_{j}$, the governing equations can be obtained by Algorithm 1 . First, observe that the ratio $b / a$ from Equations (4) and (5) is a function of $\theta_{0}$ alone. That is:

$$
\frac{b}{a}=\frac{\int_{0}^{\theta_{0}} \frac{\sin (\theta) d \theta}{\sqrt{\lambda-\sin (\theta)+n \cos (\theta)}}}{\int_{0}^{\theta_{0}} \frac{\cos (\theta) d \theta}{\sqrt{\lambda-\sin (\theta)+n \cos (\theta)}}}
$$

where $n=n\left(\theta_{0}\right)$ from the rolling contact constraints (Equations (6) and (7)). Therefore, using this relationship and Equations (10) and (11), a single nonlinear equation in $\theta_{0}$ is obtained, which can be solved using a tool such as Matlab's fsolve or a custom line search. Additionally, parameters $a$ and $b$ can be found by this process. Beam active length $L$ is solved for by evaluating the integrals presented in Equation (4) or (5) (one may be used for verification) using the values of $a$ and $b$. Nondimensional parameter $\alpha_{0}$ and force $P$ is determined by evaluating the integral in Equation (3) with the value found of $\theta_{0}$.

This method yields two functions of interest for actuator control: Moment at the joint due the deflection of the beam $M_{b}\left(\theta_{j}, x_{r}\right)$ and thrust force $F_{b}\left(\theta_{j}, x_{r}\right)$ on the actuation mechanism mounted on link 2. Given a set of physical parameters for the beam and the links, both functions can be readily evaluated by a computer program and used in control system development.

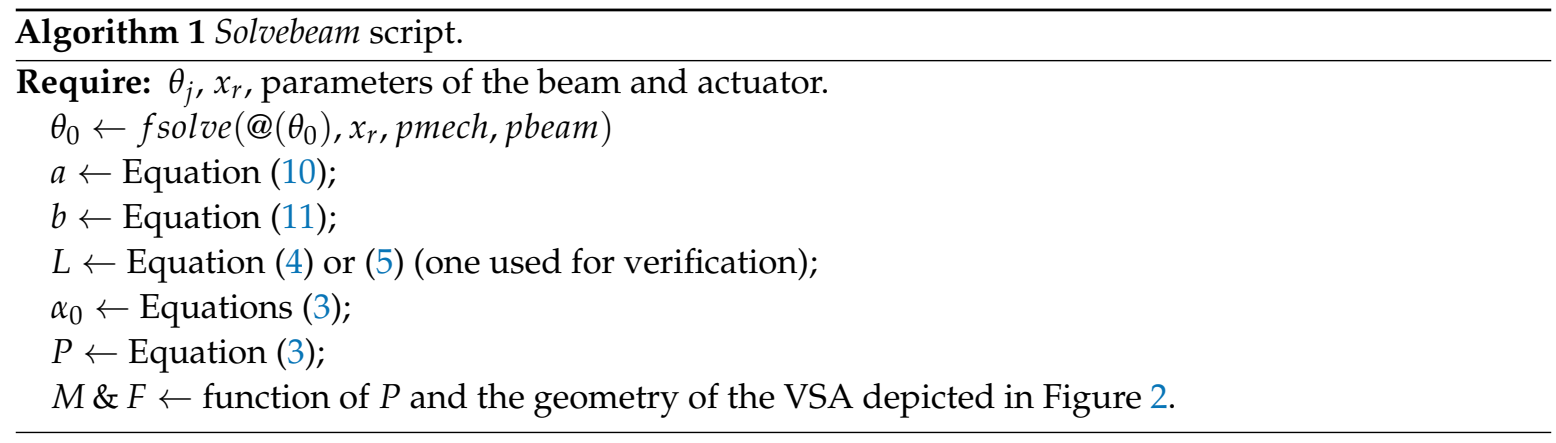

\section{Experimental Validation}

To validate the modeling approach and solution method used in this paper, a test rig was built to measure large beam deflections under the same boundary conditions as in the actuator design. As shown in Figures 4 and 5 the test rig consisted of two links connected by a joint and a cantilever beam that was fixed at Link 2 . The roller can be moved to any desired position and locked. The beam is attached as a cantilever to an L-shaped bracket that freely rotates about the joint. A cable is attached to the top arm of the L-shaped bracket and routed through a pulley. A deadweight is then applied to the end of the cable, which rotates the bracket and produces a deflection against the roller. 


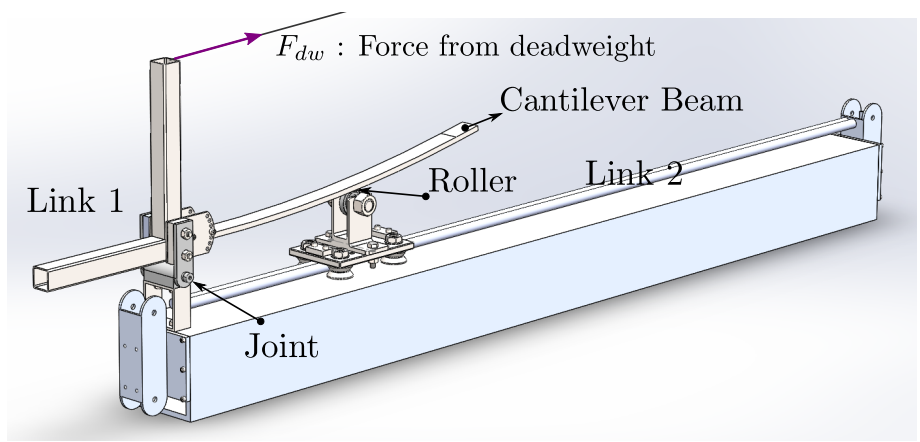

Figure 4. Schematic of test-rig structure. Cable is used to apply forces using deadweights.

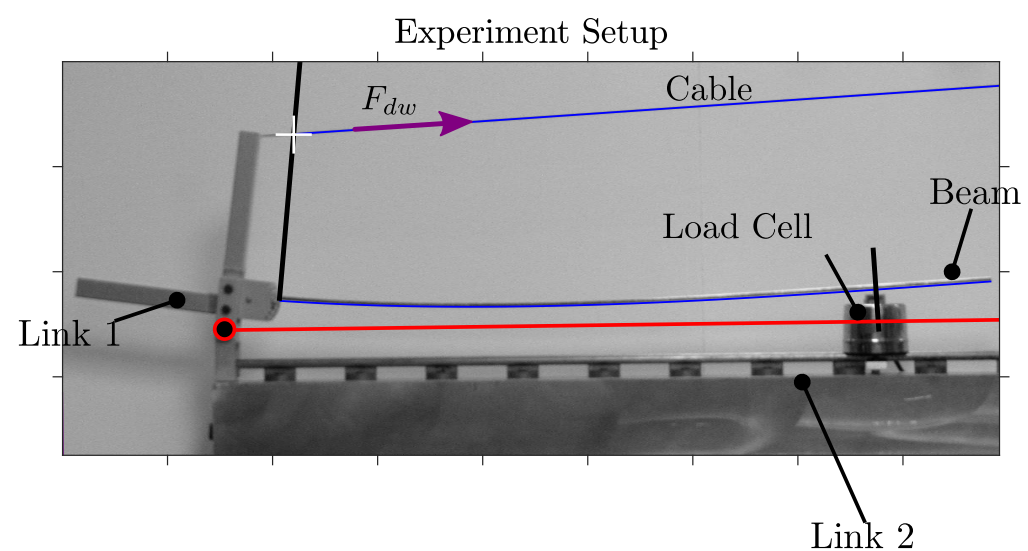

Figure 5. An instance of beam-deflection measurements with the test rig.

\section{Experiment Methodology}

A camera and image-processing software were used to capture the shape of the beam under a known load. The experiment was performed with four distinct rectangular beams with different dimensions and properties as shown in Table 1. Euler-Bernoulli theory remains accurate for sufficiently slender beams, that is, beams whose thickness is much smaller than their width and length [34]. Although a universal threshold for this ratio is not available for all situations, a lower limit of 5.4 for the length-to-thickness ratio has been mentioned [35] for cantilever beams. Table 2 shows the ratios for the active lengths involved in the experiments, largely meeting this threshold. The validity of the Euler-Bernoulli assumptions is best supported by the accuracy of the experiment predictions.

The elastic modulus of each beam was estimated using free-oscillation experiments [36] and direct measurements of cross-section properties and beam density. A set of known weights were applied to the cable, with the roller position locked. As a result, the beam underwent deflection, resulting in a reaction force from roller $F$. Dimensions $a, b$, and $e$ were extracted by image processing. Beam shape was estimated by fitting a third-degree polynomial function to image points. Other geometric properties of the beam were estimated by linear fitting and by direct measurements from the test rig.

Table 1. Beam parameters.

\begin{tabular}{cccc}
\hline Material & Cross-Section $(w \times h)(\mathbf{m m})$ & Mass $\mathbf{( k g )}$ & E (MPa) \\
\hline 6061 aluminum & $5.46 \times 31.70$ & 0.3823 & 70.8 \\
6061 aluminum & $5.46 \times 25.49$ & 0.4044 & 63.1 \\
1075 spring steel & $3.16 \times 25.45$ & 0.5646 & 194.0 \\
Low-carbon steel & $4.69 \times 25.20$ & 0.8440 & 169.2 \\
\hline
\end{tabular}


Table 2. Width-to-length and thickness-to-length ratios for the two active lengths used in the experiments $\left(l_{1}=0.75 \mathrm{~m}\right.$ and $\left.l_{2}=0.43 \mathrm{~m}\right)$.

\begin{tabular}{ccccc}
\hline Material & $l_{1} / w$ & $l_{1} / h$ & $l_{2} / w$ & $l_{2} / h$ \\
\hline 6061 aluminum & 23.7 & 137.4 & 13.6 & 78.8 \\
6061 aluminum & 29.4 & 137.4 & 16.9 & 78.8 \\
1075 spring steel & 29.5 & 237.3 & 16.9 & 136.1 \\
Low-carbon steel & 29.8 & 159.9 & 17.1 & 91.7 \\
\hline
\end{tabular}

From Figure 6, contact force $P$ can be written as the following equation:

$$
P=\frac{m g e+\left(x_{d}+d_{2}\right) m g \sin (\alpha)+\left(y_{d}+d_{1}\right) m g \cos (\alpha)}{n x_{d}+y_{d}+a+b n}
$$

The above equation contains the mass of Link 1 , intended to account for the gravitational moment due to the weight of Link 1 . However, this influence was subsequently determined to be insignificant. Aiming to validate Equation (13), the roller structure was replaced by a load cell (Omega Engineering, Norwalk, CT, USA). The difference between experiment results and actual force $P$ was small across all tests. Specifically, an overall root mean square (RMS) of $1.956 \mathrm{~N}$ was found using four beams and three materials according to the following formula:

$$
P_{R M S}=\sqrt{\frac{\sum_{n=1}^{N}\left|P_{n}\right|^{2}}{N}}
$$

where $N$ is the total number of trials and $P_{n}$ is the difference between forces $P_{\exp }$ extracted experimentally and forces $P_{\text {alg }}$ calculated by the proposed algorithm. A total of $N=56$ trials were performed, with contact forces varying between 2 and $20 \mathrm{~N}$.

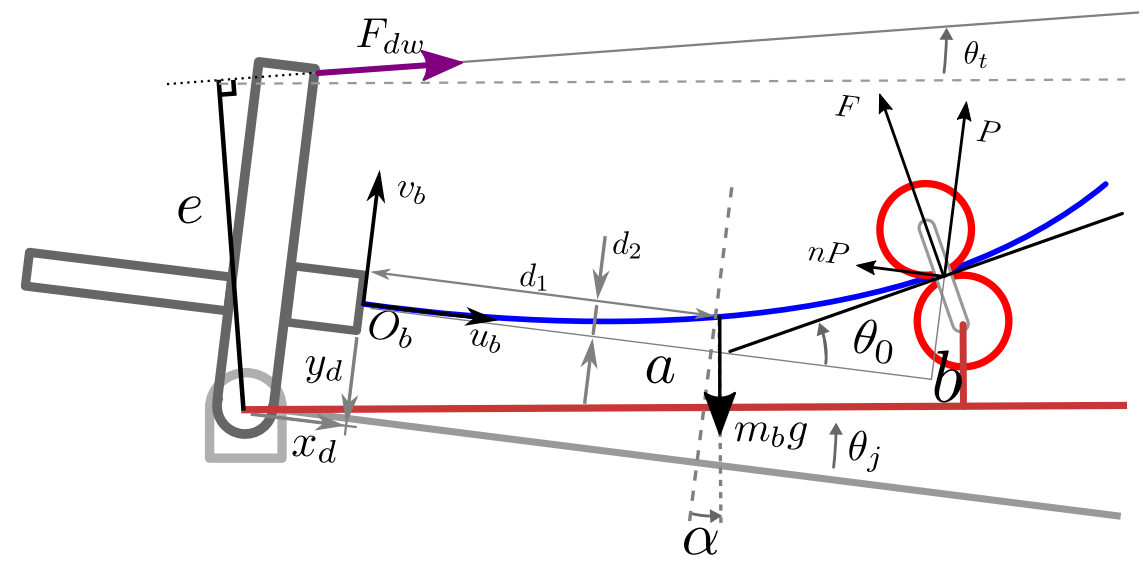

Figure 6. Geometry and loading diagram of the test rig used to derive contact force between roller and beam.

The accuracy of the proposed approach can also be evaluated using nondimensional parameter $\alpha_{0}$ from Equation (15). For this, theoretical reaction $P$ was found for each $\alpha_{0}$ calculated with experimental data. These theoretical forces were compared with predictions using the algorithm and forces extracted from the experiment using image processing and static analysis, as described earlier.

The results of these comparisons are presented in Figures 7 and 8 for $L=0.750 \mathrm{~m}$ and $L=0.430 \mathrm{~m}$, respectively. In each figure, solid lines are calculated with the proposed algorithm. RMS errors between measurements and calculations for each beam were 1.1525, 2.9869, 0.92602, and $1.8255 \mathrm{~N}$, indicating excellent agreement. Beam dimensions were selected to cover a wide range of nondimensional parameter $\alpha_{0}$. This ensures that the validity of the proposed calculation method is not restricted to 
the relatively small dimensional range of the specimens used in the experiment. It is expected that slender beams with arbitrary dimensions under loading magnitudes matching the range of $\alpha_{0}$ result in similarly accurate predictions.

$$
P_{\text {theo }} \triangleq\left(\frac{\alpha_{0}}{L}\right)^{2} \frac{E I}{2}
$$

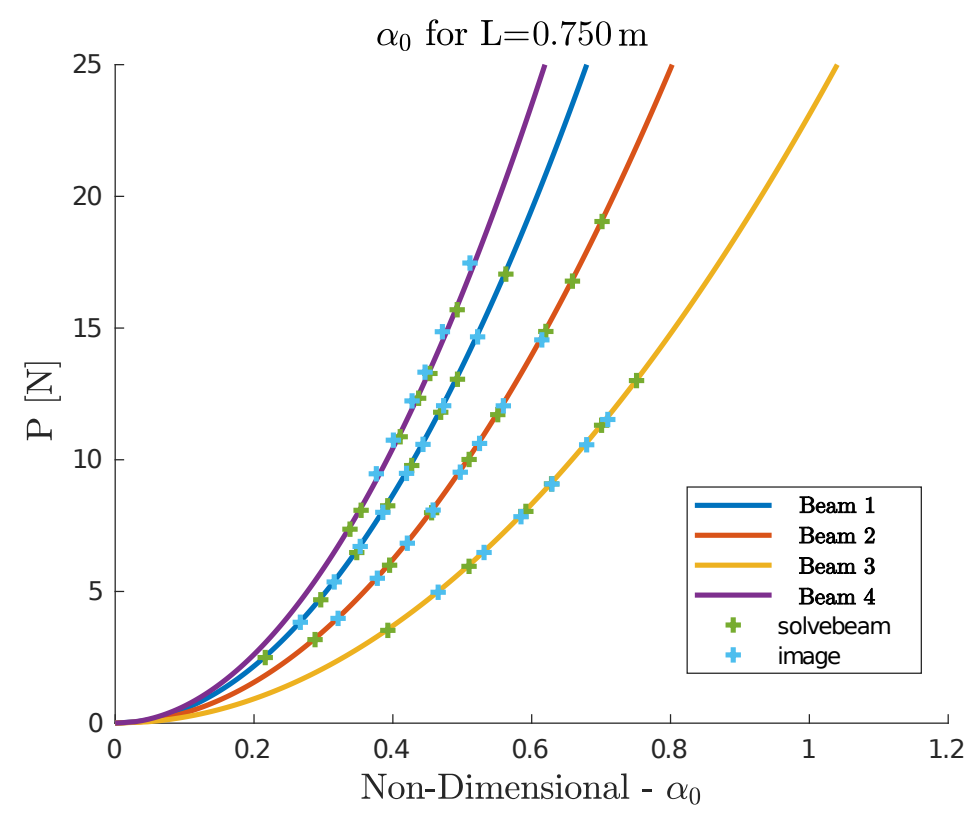

Figure 7. Contacted force $P$ versus nondimensional parameter for beam length of $0.75 \mathrm{~m}$.

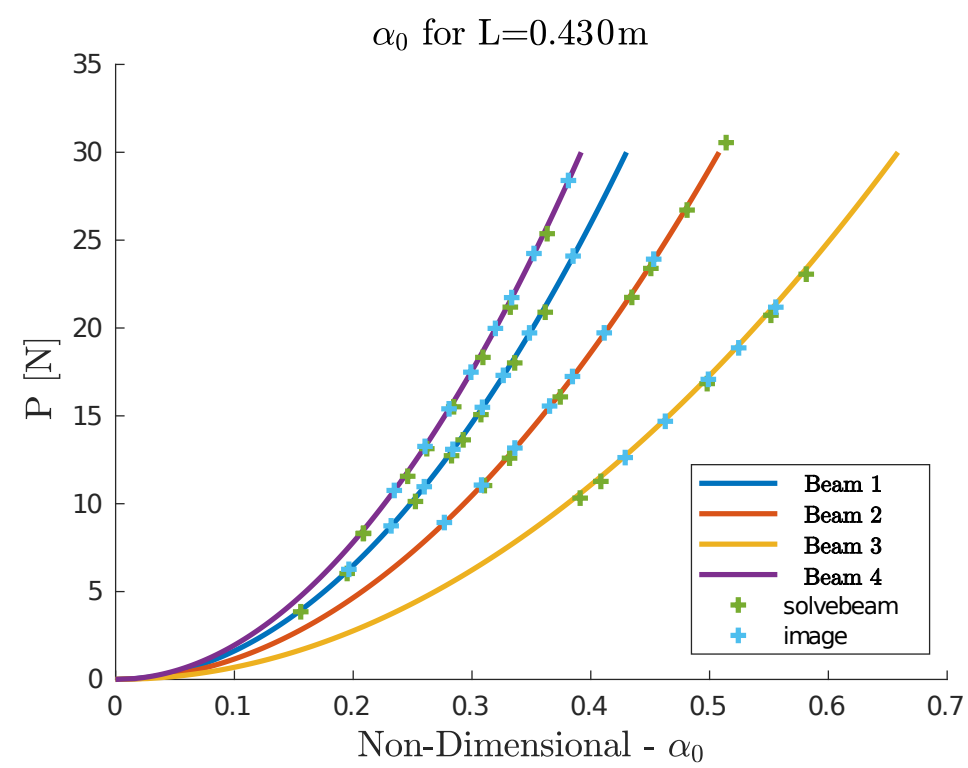

Figure 8. Contacted force $P$ versus nondimensional parameter for beam length of $0.43 \mathrm{~m}$.

\section{Conclusions and Future Work}

A new design for an actuation mechanism with controllable stiffness was presented. A formulation of large beam deflections with a rolling contact constraint was developed, and a numerical solution 
algorithm was established. Experimental procedures using a custom test rig validated the formulation and solution algorithm.

A marked advantage of the proposed design over previous VSA concepts is that the used mechanism to vary stiffness does not incur significant power losses, but rather is designed to convert and store the energy released by the beam in electric form by means of a regenerative drive system containing a storage element such as a supercapacitor. Moreover, damping effects can be introduced by means of control without the intervention of lossy physical damping elements.

The proposed mechanism uses a straight beam that may reduce the applicability of the concept in prosthetic legs due to a range of motion limitations. The cantilever beam may exhibit high root stresses and protrude inconveniently when large link rotations are attempted. One way to mitigate this limitation is to consider curved or variable-section beams that can be optimally designed. The method of this paper would require substantial modifications to allow for such variable geometries. The proposed VSA may be more conveniently used as an orthotic device around human joints having smaller ranges of motion, for instance, for ankle or wrist dorsiflexion.

The proposed calculation method requires the solution of a nonlinear equation in which numerical integrations are involved. However, model-based real-time control algorithms may require faster computations. A possible solution is to use the proposed method offline to generate moment data for a range of roller positions and link angles. The control algorithm may then access the solutions in the form of a look-up table.

Further work related to the VSA of this paper may involve optimization of the shape and material of the beam, including the possibility of using functionally graded designs. Direct nonlinear control techniques could be explored that achieve impedance regulation in combination with energy-optimization methods.

Author Contributions: Conceptualization, H.R.; Formal analysis, E.G.d.S.; Investigation, E.G.d.S.; Methodology, E.G.d.S. and H.R.; Software, E.G.d.S.; Validation, E.G.d.S.; Writing—original draft, E.G.d.S.; Writing一review and editing, H.R.

Funding: This research was funded by the U.S. National Science Foundation, grant number 1544702.

Conflicts of Interest: The authors declare no conflict of interest. The founding sponsors had no role in the design of the study; in the collection, analyses, or interpretation of data; in the writing of the manuscript; and in the decision to publish the results.

\section{Abbreviations}

The following abbreviations are used in this manuscript:

$\begin{array}{ll}\text { VSA } & \text { Variable Stiffness Actuator } \\ \text { SEA } & \text { Series Elastic Actuation } \\ \text { DOF } & \text { Degree of Freedom } \\ \text { MIA } & \text { Mechanical Impedance Adjuster } \\ \text { LWR } & \text { Lightweight Robot } \\ \text { FEA } & \text { Finite-Element Analysis } \\ \text { PRBM } & \text { Pseudorigid-Body Model }\end{array}$

\section{Appendix A}

\section{Appendix A.1. Transformations in Homogeneous Coordinates}

End deflections $a$ and $b$ presented in Equations (10) and (11) of Section 3.2 are defined by using a set of homogeneous coordinate transformations (translation and rotation), which is presented in the following: 


$$
\begin{aligned}
\operatorname{Trans}_{x, a} & =\left[\begin{array}{llll}
1 & 0 & 0 & a \\
0 & 1 & 0 & 0 \\
0 & 0 & 1 & 0 \\
0 & 0 & 0 & 1
\end{array}\right] \\
\operatorname{Trans}_{y, b} & =\left[\begin{array}{llll}
1 & 0 & 0 & 0 \\
0 & 1 & 0 & b \\
0 & 0 & 1 & 0 \\
0 & 0 & 0 & 1
\end{array}\right] \\
\operatorname{Rot}_{z, \gamma}= & {\left[\begin{array}{cccc}
\cos \gamma & -\sin \gamma & 0 & 0 \\
\sin \gamma & \cos \gamma & 0 & 0 \\
0 & 0 & 1 & 0 \\
0 & 0 & 0 & 1
\end{array}\right] }
\end{aligned}
$$

\section{References}

1. Zinn, M.; Khatib, O.; Roth, B.; Salisbury, J.K. Playing it safe [human-friendly robots]. IEEE Rob. Autom Mag. 2004, 11, 12-21. [CrossRef]

2. Van Ham, R.; Sugar, T.G.; Vanderborght, B.; Hollander, K.W.; Lefeber, D. Compliant actuator designs. IEEE Rob. Autom. Mag. 2009, 16, 81-94.

3. Wolf, S.; Grioli, G.; Eiberger, O.; Friedl, W.; Grebenstein, M.; Höppner, H.; Burdet, E.; Caldwell, D.G.; Carloni, R.; Catalano, M.G.; et al. Variable stiffness actuators: Review on design and components. IEEE/ASME Trans. Mechatron. 2016, 21, 2418-2430. [CrossRef]

4. Vanderborght, B.; Albu-Schäffer, A.; Bicchi, A.; Burdet, E.; Caldwell, D.G.; Carloni, R.; Catalano, M.; Eiberger, O.; Friedl, W.; Ganesh, G.; et al. Variable impedance actuators: A review. Rob. Autom. Syst. 2013, 61, 1601-1614. [CrossRef]

5. Morita, T.; Sugano, S. Design and development of a new robot joint using a mechanical impedance adjuster. In Proceedings of the 1995 IEEE International Conference on Robotics and Automation, Nagoya, Japan, 21-27 May 1995; Volume 3, pp. 2469-2475.

6. Morita, T.; Sugano, S. Development of 4-DOF manipulator using mechanical impedance adjuster. In Proceedings of the 1996 IEEE International Conference on Robotics and Automation, Minneapolis, MN, USA, 22-28 April 1996; Volume 4, pp. 2902-2907.

7. Morita, T.; Sugano, S. Development and evaluation of seven DOF MIA ARM. In Proceedings of the 1997 IEEE International Conference on Robotics and Automation, Monterey, CA, USA, 10-11 July 1997; Volume 1, pp. 462-467.

8. Petit, F; Albu-Schäffer, A. State feedback damping control for a multi DOF variable stiffness robot arm. In Proceedings of the 2001 IEEE International Conference on Robotics and Automation, Seoul, Korea, 21-26 May 2001; pp. 5561-5567.

9. Verstraten, T.; Beckerle, P.; Furnémont, R.; Mathijssen, G.; Vanderborght, B.; Lefeber, D. Series and parallel elastic actuation: Impact of natural dynamics on power and energy consumption. Mech. Mach. Theory 2016, 102, 232-246. [CrossRef]

10. Nasiri, R.; Khoramshahi, M.; Shushtari, M.; Ahmadabadi, M.N. Adaptation in variable parallel compliance: Towards energy efficiency in cyclic tasks. IEEE/ASME Trans. Mechatron. 2017, 22, 1059-1070. [CrossRef]

11. Verstraten, T.; Geeroms, J.; Mathijssen, G.; Convens, B.; Vanderborght, B.; Lefeber, D. Optimizing the power and energy consumption of powered prosthetic ankles with series and parallel elasticity. Mech. Mach. Theory 2017, 116, 419-432. [CrossRef]

12. Geeroms, J.; Flynn, L.; Jimenez-Fabian, R.; Vanderborght, B.; Lefeber, D. Energetic analysis and optimization of a MACCEPA actuator in an ankle prosthesis. Auton. Robots 2018, 42, 147-158. [CrossRef]

13. Salisbury, J.K. Active stiffness control of a manipulator in cartesian coordinates. In Proceedings of the 1980 19th IEEE Conference on Decision and Control including the Symposium on Adaptive Processes, Albuquerque, NM, USA, 10-12 December 1980; Volume 19, pp. 95-100. 
14. Takakura, S.; Murakami, T.; Ohnishi, K. An approach to collision detection and recovery motion in industrial robot. In Proceedings of the 15th Annual Conference of IEEE Industrial Electronics Society (IECON'89), Philadelphia, PA, USA, 6-10 November 1989; pp. 421-426.

15. Shetty, B.R.; Ang, M.H. Active compliance control of a PUMA 560 robot. In Proceedings of the 1996 IEEE International Conference on Robotics and Automation, Minneapolis, MN, USA, 22-28 April 1996; Volume 4, pp. 3720-3725.

16. Burger, R.; Haddadin, S.; Plank, G.; Parusel, S.; Hirzinger, G. The driver concept for the DLR lightweight robot III. In Proceedings of the 2010 IEEE/RSJ International Conference on Intelligent Robots and Systems (IROS), Taipei, Taiwan, 18-22 October 2010; pp. 5453-5459.

17. Bischoff, R.; Kurth, J.; Schreiber, G.; Koeppe, R.; Albu-Schäffer, A.; Beyer, A.; Eiberger, O.; Haddadin, S.; Stemmer, A.; Grunwald, G.; et al. The KUKA-DLR Lightweight Robot arm-A new reference platform for robotics research and manufacturing. In Proceedings of the 2010 41st International Symposium on Robotics (ISR) and 2010 6th German Conference on Robotics (ROBOTIK), Munich, Germany, 7-9 June 2010; pp. 1-8.

18. De Luca, A.; Albu-Schaffer, A.; Haddadin, S.; Hirzinger, G. Collision Detection and Safe Reaction with the DLR-III Lightweight Manipulator Arm. In Proceedings of the 2006 IEEE/RSJ International Conference on Intelligent Robots and Systems, Beijing, China, 9-15 October 2006; pp. 1623-1630.

19. Fuchs, S.; Haddadin, S.; Keller, M.; Parusel, S.; Kolb, A.; Suppa, M. Cooperative bin-picking with time-of-flight camera and impedance controlled DLR Lightweight Robot III. In Proceedings of the 2010 IEEE/RSJ International Conference on Intelligent Robots and Systems (IROS), Taipei, Taiwan, 18-22 October 2010; pp. 4862-4867.

20. Wang, W.; Loh, R.N.; Gu, E.Y. Passive compliance versus active compliance in robot-based automated assembly systems. Ind. Robot 1998, 25, 48-57. [CrossRef]

21. Dahiya, A.; Braun, D.J. Efficiently tunable positive-negative stiffness actuator. In Proceedings of the 2017 IEEE International Conference on Robotics and Automation (ICRA), Singapore, 29 May-3 June 2017; pp. 1235-1240.

22. Braun, D.J.; Apte, S.; Adiyatov, O.; Dahiya, A.; Hogan, N. Compliant actuation for energy efficient impedance modulation. In Proceedings of the 2016 IEEE International Conference on Robotics and Automation (ICRA), Stockholm, Sweden, 16-21 May 2016; pp. 636-641.

23. Khalaf, P.; Warner, H.; Hardin, E.; Richter, H.; Simon, D. Development and experimental validation of an energy regenerative prosthetic knee controller and prototype. In Proceedings of the ASME 2018 Dynamic Systems and Control Conference, Atlanta, GA, USA, 27-29 June 2018.

24. Shepherd, M.K.; Rouse, E.J. The VSPA foot: A quasi-passive ankle-foot prosthesis with continuously variable stiffness. IEEE Trans. Neural Syst. Rehabil. Eng. 2017, 25, 2375-2386. [CrossRef] [PubMed]

25. Shepherd, M.K.; Rouse, E.J. Design of a quasi-passive ankle-foot prosthesis with biomimetic, variable stiffness. In Proceedings of the 2017 IEEE International Conference on Robotics and Automation (ICRA), Singapore, 29 May-3 June 2017; pp. 6672-6678.

26. Bisshopp, K.; Drucker, D. Large deflection of cantilever beams. Q. Appl. Math. 1945, 3, 272-275. [CrossRef]

27. Zhang, A.; Chen, G. A comprehensive elliptic integral solution to the large deflection problems of thin beams in compliant mechanisms. J. Mech. Rob. 2013, 5, 021006. [CrossRef]

28. Campanile, L.; Hasse, A. A simple and effective solution of the elastica problem. Proc. Ins. of Mech. Eng. C J. Mech. Eng. Sc. 2008, 222, 2513-2516. [CrossRef]

29. Mattiasson, K. Numerical results from large deflection beam and frame problems analysed by means of elliptic integrals. Int. J. Numer. Methods Eng. 1981, 17, 145-153. [CrossRef]

30. Saxena, A.; Kramer, S. A simple and accurate method for determining large deflections in compliant mechanisms subjected to end forces and moments. J. Mech. Des. 1998, 120, 392-400. [CrossRef]

31. Banerjee, A.; Bhattacharya, B.; Mallik, A. Large deflection of cantilever beams with geometric non-linearity: Analytical and numerical approaches. Int. J. Non Linear Mech. 2008, 43, 366-376. [CrossRef]

32. dos Santos, E.G.; Richter, H. Modeling and Control of a Novel Variable-Stiffness Regenerative Actuator. In Proceedings of the ASME 2018 Dynamic Systems and Control Conference, Atlanta, GA, USA, 27-29 June 2018; V002T24A003.

33. Spong, M.W.; Hutchinson, S.; Vidyasagar, M. Robot Modeling and Control; John Wiley \& Sons, Inc.: New York, NT, USA, 2006; Volume 3. 
34. Bauchau, O.A.; Craig, J.I. Structural Analysis: With Applications to Aerospace Structures; Volume 163; Springer: Dordrecht, The Netherlands, 2009.

35. Troughton, M. Handbook of Plastics Joining: A Practical Guide; William Andrew: Norwich, NY, USA, 2008.

36. Genta, G.; Delprete, C. Some considerations on the experimental determination of moments of inertia. Meccanica 1994, 29, 125-141. [CrossRef]

(C) 2019 by the authors. Licensee MDPI, Basel, Switzerland. This article is an open access article distributed under the terms and conditions of the Creative Commons Attribution (CC BY) license (http:/ / creativecommons.org/licenses/by/4.0/). 